\title{
Computing the magnetic field response of the proton
}

\author{
Ryan Bignell ${ }^{1, *}$, Waseem Kamleh $^{1, * *}$, and Derek Leinweber ${ }^{1, * * *}$ \\ ${ }^{1}$ The Special Research Centre for the Subatomic Structure of Matter (CSSM) \\ Department of Physics, University of Adelaide, Adelaide, South Australia 5005, Australia
}

\begin{abstract}
Background field methods offer an approach through which fundamental non-perturbative hadronic properties can be studied. Lattice QCD is the only ab initio method with which Quantum Chromodynamics can be studied at low energies; it involves numerically calculating expectation values in the path integral formalism. This requires substantial investment in high performance supercomputing resources. A particular challenge of lattice QCD is isolating the desired state, rather than a superposition of excited states. While extensive work has been performed which allows the ground state to be identified in lattice QCD calculations, this remains a challenging proposition for the ground state in the presence of a uniform magnetic field field. Quark level projection operators are introduced to resolve this challenge and thus allow for extraction of the magnetic polarisability.
\end{abstract}

\section{Introduction}

The response of the proton to an external magnetic field is described by the magnetic polarisability. Measurement of this quantity is difficult $[1,2]$ but experimental measurements in recent years have yielded improved results [3-5]. The numerical ab initio method for nonperturbative QCD known as lattice QCD can provide valuable insight and predictions.

The magnetic polarisability is accessed using lattice QCD through the introduction of a uniform background field [6-8] which results in the energy-field relation [7, 9-14]

$$
E(B)=m+\vec{\mu} \cdot \vec{B}+(2 n+1) \frac{|q e B|}{2 m}-\frac{4 \pi}{2} \beta B^{2}+\cdots
$$

where the proton has mass $m$, magnetic moment $\vec{\mu}$, and magnetic polarisability $\beta$. The term proportional to $|q e B|$ is the Landau energy [15] of the proton, where in principle there is an infinite tower of Landau levels for $n=0,1,2, \ldots$. Naively the linear and quadratic coefficients of Eq. (1) could be fit and thus the magnetic polarisability extracted. However this approach is insufficient as the contribution of the magnetic polarisability to the proton energy is small compared to the overall proton energy. The magnetic polarisability appears at second order in the energy expansion.

When a background field is present, three dimensional gauge-covariant Gaussian smearing fails to efficiently isolate the proton ground state in contrast to pure QCD calculations

\footnotetext{
*e-mail: ryan.bignell@adelaide.edu.au

**e-mail: waseem.kamleh@adelaide.edu.au

***e-mail: derek.leinweber@adelaide.edu.au
} 
[16]. The magnetic field introduces electromagnetic perturbations into the dynamics of the charged quarks and breaks three-dimensional spatial symmetry, thus altering the physics present. In the absence of QCD interactions, each quark will have a Landau energy which is proportional to its charge; while in the presence of QCD the quarks hadronise such that, in the confining phase, the Landau energy corresponds to that of the composite hadron.

While the competition in the confining phase between the magnetic and QCD interactions is clear, previous studies have demonstrated that Landau physics remains relevant when QCD interactions are present $[10,17]$. This inspires the development of lattice quark operators that capture both of these forces. We construct lattice two-point correlation functions which have improved overlap with the energy eigenstates of the proton in a background magnetic field by choosing asymmetric source and sink operators.

Our previous study of the neutron [10] explored a quark sink projection formed using the eigenmodes of the two-dimensional $U(1)$ lattice Laplacian. These are associated with the Landau modes of a charged particle in a magnetic field. Here a projection derived from the eigenmodes of the two-dimensional lattice $S U(3) \times U(1)$ Laplacian operator is considered. We consider a fully gauge-covariant eigenmode-projected quark sink, encapsulating both QCD and Landau level physics which eliminates the need for gauge fixing. We find that the quark projection operator formed from the $S U(3) \times U(1)$ modes is effective in isolating the proton ground state in an external magnetic field. This enables an accurate determination of the magnetic polarisability of the proton for the first time.

\section{Background Field Method}

The background field method is utilised to introduce a constant magnetic field along the $\hat{z}$ axis. We derive the technique first in the continuum where a minimal electromagnetic coupling is added to form the covariant derivative

$$
D_{\mu}=D_{\mu}^{\mathrm{QCD}}+i q e A_{\mu},
$$

where $A_{\mu}$ is the electromagnetic four potential and $q e$ is the charge of the fermion field. The equivalent modification on the lattice is to multiply the QCD gauge links by an exponential phase factor

$$
U_{\mu}(x) \rightarrow U_{\mu}(x)^{(B)}=\mathrm{e}^{i a q e A_{\mu}(x)} U_{\mu}(x),
$$

where $a$ is the lattice spacing. These modified gauge links are used in the lattice QCD simulation.

As $\vec{B}=\vec{\nabla} \times \vec{A}$, a uniform magnetic field along the $\hat{z}$ axis is obtained by exploiting both $A_{x}$ and $A_{y}$. We set $A_{x}=-B y$ throughout the lattice. The constant magnetic field is maintained across the $\hat{y}$ edge of the lattice, where periodic boundary conditions are in effect, by setting $A_{y}=+B N_{y} x$ along the $\hat{y}$ boundary $y=a N_{y}$. A quantisation condition on the magnetic field strength is thus induced [14]

$$
\text { qe } B a^{2}=\frac{2 \pi k}{N_{x} N_{y}}
$$

where $N_{x}$ and $N_{y}$ are the spatial dimensions of the lattice and $k$ is an integer which specifies the field strength in terms of the minimum field strength quanta.

In this work, the field quanta $k$ is in terms of the charge of the down quark, i.e., $k=k_{d}$ and $q=q_{d}=-1 / 3$. Hence a field with $k_{d}=1$ is in the $-\hat{z}$ direction and the magnetic field experienced by the proton is defined to be $k_{B}=-3 k_{d}$.

In order to remove the additive mass renormalisation due to the Wilson term with a uniform background field [18, 19], the Background-Field-Corrected-Clover action [20] is used. 


\section{Quark Operators}

Asymmetric source and sink operators are used, enabling the construction of proton correlation functions which have good overlap with the lowest lying energy eigenstate of the proton in an external magnetic field.

\section{1 $\mathrm{SU}(3) \times \mathrm{U}(1)$ eigenmode projection}

We construct a fully gauge covariant quark sink projection operator that encapsulates both QCD and electromagnetic influences by calculating the low-lying eigenmodes $\left|\psi_{i}\right\rangle$ of the two-dimensional lattice Laplacian

$$
\Delta_{\vec{x}, \vec{x}^{\prime}}=4 \delta_{\vec{x}, \vec{x}^{\prime}}-\sum_{\mu=1,2} U_{\mu}(\vec{x}) \delta_{\vec{x}+\hat{\mu}, \vec{x}^{\prime}}+U_{\mu}^{\dagger}(\vec{x}-\hat{\mu}) \delta_{\vec{x}-\hat{\mu}, \vec{x}^{\prime}},
$$

where $U_{\mu}(\vec{x})$ are the full $S U(3) \times U(1)$ gauge links applied in the lattice QCD simulation. A projection operator focusing on the low-energy physics is defined by truncating the completeness relation

$$
1=\sum_{i}\left|\psi_{i}\right\rangle\left\langle\psi_{i}\right|
$$

In the pure $U(1)$ case, the lattice Landau levels are eigenmodes of the (2D) $U(1)$ Laplacian ( as defined in Eq. (5) but for $U(1)$ gauge links only ). In this limit, the lowest Landau level on the lattice has a degeneracy equal to the magnetic field quanta $|k|$ in Eq. (4) which provides a natural place to truncate the completeness relation. Introducing QCD interactions into the Laplacian causes the $U(1)$ modes associated with the different Landau levels to mix such that it is no longer possible to clearly distinguish the modes which are associated with the lowest Landau level at small field strengths.

As such we select a fixed number $n>|k|$ of the lowest modes to project. The truncation has a similar effect to two-dimensional smearing, by filtering out the high frequency modes. A small number of modes $n$ increases the noise in the projected hadron correlator as large amounts of sink smearing does. By inspection of varying eigenmode number, $n=96$ eigenmodes is chosen.

The low-lying eigenspace is calculated independently for each $(z, t)$-slice on the lattice due to the two-dimensional nature of the Laplacian. Thus the four-dimensional coordinate space representation of an eigenmode

$$
\left\langle\vec{x}, t \mid \psi_{i, \vec{B}}\right\rangle=\psi_{i, \vec{B}}(x, y \mid z, t),
$$

can be interpreted as selecting the two-dimensional coordinate space representation $\psi_{i, \vec{B}}(x, y)$ from the eigenspace which belongs to the corresponding $(z, t)$-slice of the lattice. Hence the four-dimensional coordinate space representation of the projection operator is

$$
P_{n}\left(\vec{x}, t ; \vec{x}^{\prime}, t^{\prime}\right)=\sum_{i=1}^{n}\left\langle\vec{x}, t \mid \psi_{i, \vec{B}}\right\rangle\left\langle\psi_{i, \vec{B}} \mid \vec{x}^{\prime}, t^{\prime}\right\rangle \delta_{z z^{\prime}} \delta_{t t^{\prime}} .
$$

This projection operator is then applied to the the quark propagator at the sink in a coordinate space representation as

$$
S_{n}(\vec{x}, t ; \overrightarrow{0}, 0)=\sum_{\vec{x}^{\prime}} P_{n}\left(\vec{x}, t ; \vec{x}^{\prime}, t\right) S\left(\vec{x}^{\prime}, t ; \overrightarrow{0}, 0\right) .
$$



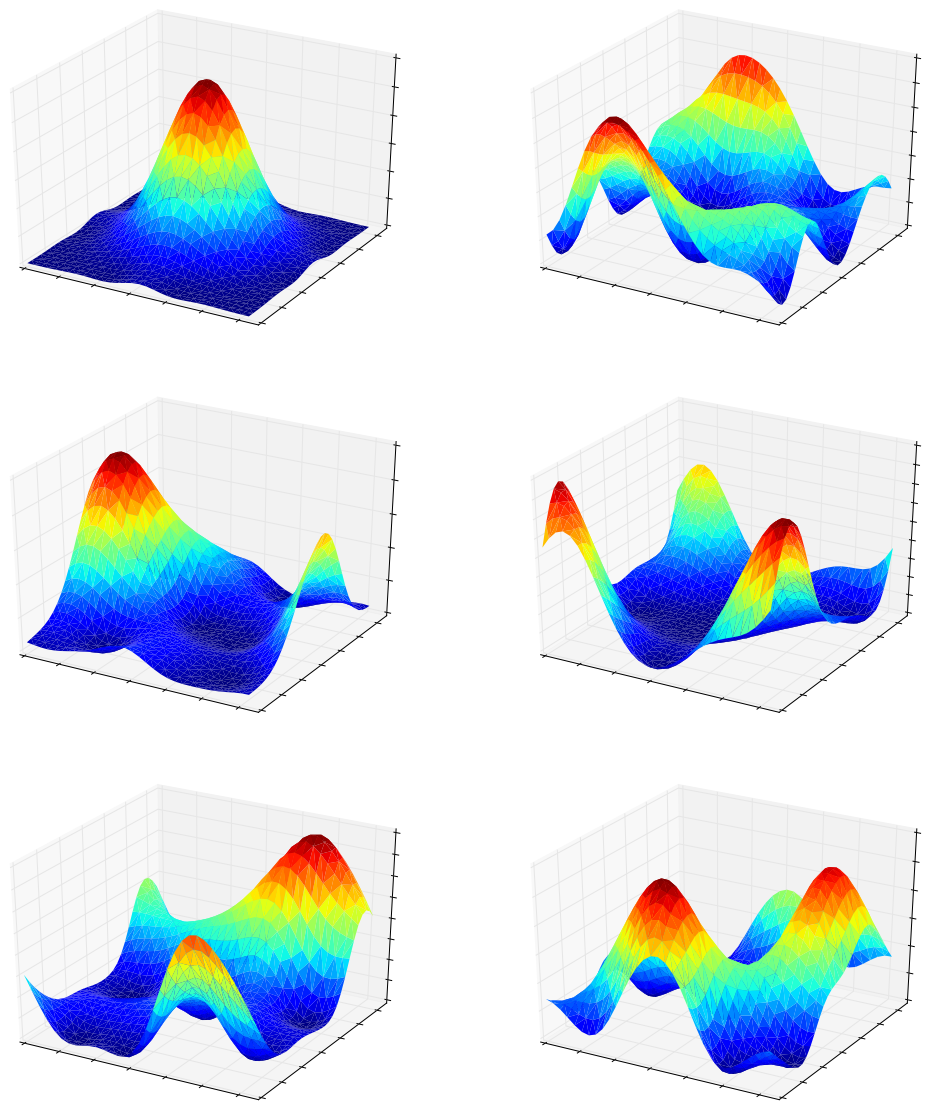

Figure 1. Lowest-lying pure U(1) eigenmode probability densities of the lattice Laplacian operator in a constant background magnetic field oriented in the $\hat{z}$ direction are plotted as a function of the $x, y$ coordinates. The origin is at the centre of the $x-y$ plane. As $k_{B}=-3 k_{d}$, the degenerate eigenmodes for the sixth quantised field strength relevant to the proton for $k_{d}=2$ are displayed in a linear combination that maximises the overlap of the first mode with the source.

\subsection{Source Smearing}

A smeared source is tuned to isolate the QCD ground state. A broad range of smearing levels are examined at zero field strength, $B=0$ in order to do this. The $S U(3) \times U(1)$ eigenmode projection technique is used at the sink in order to match the non-zero field simulations. Smearing levels of $N_{s m}=150,175,300$ and 250 are used for masses $m_{\pi}=702,570,411$ and $296 \mathrm{MeV}$ respectively [21].

\subsection{Hadronic Landau Projection}

While the $S U(3) \times U(1)$ eigenmode projection technique defined above is relevant to Landau level effects at the quark level, the proton will also experience Landau level behaviour at a hadronic level as it is a charged hadron. A standard three-dimensional Fourier projection of the two-point correlation function is not appropriate for the proton in an external magnetic 
field as the presence of the background field causes the energy eigenstates of the charged proton to no longer be eigenstates of the $p_{x}$ and $p_{y}$ momentum components. As such, we use the eigenmodes of the $U(1)$ lattice Laplacian which have a well defined degeneracy for the lowest lattice Landau level $[10,22]$. We take linear combinations of the degenerate modes designed to maximise overlap of the first mode with the smeared source. Denoting the Landau-level index, $i$, by $k_{B}, j$ where $j$ spans the $\left|k_{B}\right|$ degenerate eigenmodes, we project onto the lowest proton Landau level explicitly while selecting a specific value for the $z$ component of momentum

$$
G\left(p_{z}, \vec{B}, t\right)=\sum_{\vec{r}} \sum_{j=1}^{\left|k_{B}\right|} \psi_{k_{B}, j, \vec{B}}(x, y) \mathrm{e}^{-i p_{z} z}\langle\Omega|T\{\chi(\vec{r}, t) \bar{\chi}(\overrightarrow{0}, t)\}| \Omega\rangle,
$$

where $\vec{r}=(x, y, z)$. The degeneracy of the lowest-lying Landau mode is given by the magnetic-field quanta $\left|k_{B}\right|=\left|3 k_{d}\right|$.

In evaluating Eq. (10), we also consider the case of only considering the first degenerate mode, such that only the eigenmode having the best overlap with the source is considered.

In most cases the results are almost indistinguishable and we proceed with the sum over degenerate modes. The only exception is for the ensemble with $m_{\pi}=411 \mathrm{MeV}$. Here the first mode alone provides superior results. Figure 1 illustrates the six degenerate modes associated with the pure $U(1)$ lattice Laplacian for $k_{d}=2$, i.e. $\left|k_{B}\right|=6$.

More generally, this hadronic eigenmode-projected correlator offers superior isolation of the ground state for the proton [13] and is crucial for the identification of constant plateaus in the energy shift of Eq. (15).

\section{Magnetic Polarisability}

As the naive process of fitting to Eq. (1) as a function of field strengths is not a viable method to extract the magnetic polarisability, we instead form correlated ratios of two-point correlation functions which isolate the energy shift due to the magnetic polarisability. We define the spin-field aligned correlation function

$$
G_{1 \uparrow}(B)=G(+s,+B)+G(-s,-B),
$$

and the spin-field antialigned correlator by

$$
G_{1 \downarrow}(B)=G(+s,-B)+G(-s,+B),
$$

where spin-up/down is represented by $(+s /-s)$ respectively and the magnetic field orientation along the spin quantisation direction, $\hat{z}$, by $( \pm B)$.

The ratio required to isolate the magnetic polarisability term of Eq. (1) is

$$
R(B, t)=\frac{G_{1 \uparrow}(B, t) G_{1 \downarrow}(B, t)}{G(0, t)^{2}},
$$

where $G(0, t)$ is the spin-averaged zero-field correlator which subtracts the mass term from the total energy of the antialigned and aligned contributions. The magnetic moment contribution is removed by the product of the spin-field antialigned and aligned correlators. The desired effective energy shift is

$$
\delta E(B, t)=\frac{1}{2} \frac{1}{\delta t} \log \left(\frac{R(B, t)}{R(B, t+\delta t)}\right),
$$

and in the large Euclidean time limit this is

$$
\delta E(B, t \rightarrow \infty)=(2 n+1) \frac{|q e B|}{2 m}-\frac{4 \pi}{2} \beta B^{2}+\ldots
$$




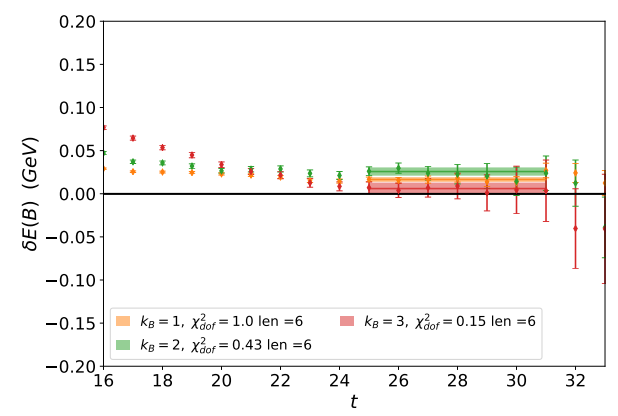

(a) The magnetic polarisability effective energy shift of Eq. (15) for the $m_{\pi}=570 \mathrm{MeV}$ proton as a function of Euclidean time (in lattice units), using a smeared source and the $S U(3) \times U(1)$ quark-eigenmode projection technique. Results for field strengths $k_{d}=1,2,3$ are shown. The selected fits for this ensemble and the $\chi_{d o f}^{2}$ are also illustrated.

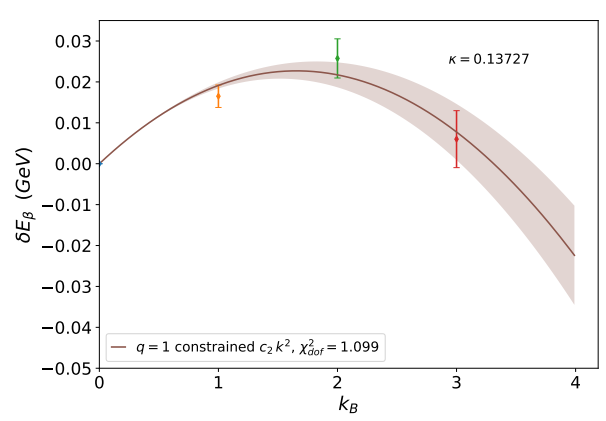

(b) Linearly constrained quadratic fit of Eq. (16) for the $m_{\pi}=570 \mathrm{MeV}$ proton.

Figure 2. Effective energy shift Euclidean-time fits (a) and the linearly constrained quadratic fit for the $m_{\pi}=570 \mathrm{MeV}$ proton (b).

\subsection{Fitting}

For the proton, we primarily consider the linearly constrained energy shift

$$
\delta E\left(k_{d}, t\right)-\frac{e B}{2 m}=c_{2} k_{d}^{2}=-\frac{4 \pi}{2} \beta B^{2}+O\left(B^{4}\right)
$$

where the lowest lying Landau level assumption has been made and the known linear term explicitly subtracted with $q=1$ such that only a term which is quadratic in $B$ is fitted. This constrains the proton to have charge $q=1$. Here we fit as a function of the integer magnetic flux quanta, $k_{d}$ and $c_{2}$ is a fit parameter which has the units of $\delta E\left(k_{d}, t\right)$.

The fit parameter $c_{2}$ and the quantisation condition of Eq. (4) provides

$$
\beta=-2 c_{2} \alpha q_{d}^{2} a^{4}\left(\frac{N_{x} N_{y}}{2 \pi}\right)^{2},
$$

where $\alpha=1 / 137 \ldots$ is the fine structure constant.

In order to fit Eq. (16), we select energy shifts at all field strengths that obey the single state ansatz which requires that a constant plateau fit can be found as a function of Euclidean lattice time $t$. The full covariance matrix $\chi_{d o f}^{2}$ is estimated using the jackknife method [23] and accounts for correlations between adjacent Euclidean times. When performing both plateau fits and fits to Eq. (16) we require that the reported result has an acceptable $\chi_{d o f}^{2}$.

\section{Results}

Hadronic Landau-projected correlation functions for the proton are calculated using the $S U(3) \times U(1)$ quark projection technique and a smeared source for several non-zero field strengths. This process ensures that both quark and hadronic Landau level effects are considered in order to isolate the proton ground state energy in an external magnetic field. The tuned smeared source provides a good representation of the QCD ground state. 
Table 1. Lattice magnetic polarisability values for the proton at each quark mass considered. The numbers in parentheses describe statistical uncertainties.

\begin{tabular}{lll}
\hline$\kappa_{u d}$ & $m_{\pi}(\mathrm{MeV})$ & $\beta\left(\times 10^{-4} \mathrm{fm}^{3}\right)$ \\
\hline 0.13700 & 702 & $1.90(19)$ \\
0.13727 & 570 & $1.87(18)$ \\
0.13754 & 411 & $1.98(21)$ \\
0.13770 & 296 & $1.93(22)$ \\
\hline
\end{tabular}

Figure 2a demonstrates the effectiveness of this approach for the $m_{\pi}=570 \mathrm{MeV}$ proton energy shift of Eq. (15). The effective energy shifts have good plateau behaviour across all field strengths considered. This behaviour is seen across each quark mass considered. For the lightest quark mass, with $m_{\pi}=296 \mathrm{MeV}$ only the first two non-zero field strengths are considered as the third is prohibitively noisy. This is the first time that plateau behaviour has been evident in the polarisability energy shift for the proton and is a consequence of our detailed approach using the $S U(3) \times U(1)$ quark projection technique along with a hadronic Landau projection.

The linearly constrained fit function of Eq. (16) along with Eq. (17) is used to determine the magnetic polarisability from the energy shifts. An example of these fits is shown in Figure $2 \mathrm{~b}$ where the fit describes the data well. Similar fit behaviour is observed at each quark mass considered.

The resulting magnetic polarisability values for the proton at each quark (pion) mass are presented in Table 1 and displayed in Figure 3 alongside a number of recent experimental results which are offet from the physical point for clarity. Reasonable agreement is seen without accounting for electro-quenching, finite-volume and the heavier-than physical quark mass. These systematics along with a chiral extrapolation to the physical masses of nature are addressed in Ref. [21].

\section{Conclusion}

The magnetic polarisability of the proton has been calculated using lattice QCD with asymmetric operators at the source and sink for the first time. The dominant QCD dynamics are captured using gauge invariant Gaussian smearing at the source while the $S U(3) \times U(1)$ eigenmode quark projection technique encodes the low-lying quark level Landau physics due to the presence of a uniform magnetic field. At the hadronic level, a Landau wave function projection onto the proton two-point correlation functions is used. The combination of these techniques has enabled constant Euclidean time fits to be found in the magnetic-polarisability energy shift of the proton for the first time.

A comphrensive investgiation of the proton using these techniques is presented in Ref. [21] where we also perform a chiral effective field theory analysis in order to account for electroquenching and finite-volume effects along with an extrapolation to the physical quark masses of nature. Thus connection with the experimental regime is achieved. The $S U(3) \times U(1)$ eigenmode quark projection technique is also applicable to further elements of the hadronic spectrum such as the neutron and hyperons in general.

\section{Acknowledgements}

We thank the PACS-CS Collaboration for making their $2+1$ flavour configurations available and the ongoing support of the International Lattice Data Grid (ILDG). This work was supported with supercomputing resources provided by the Phoenix HPC service at the University 


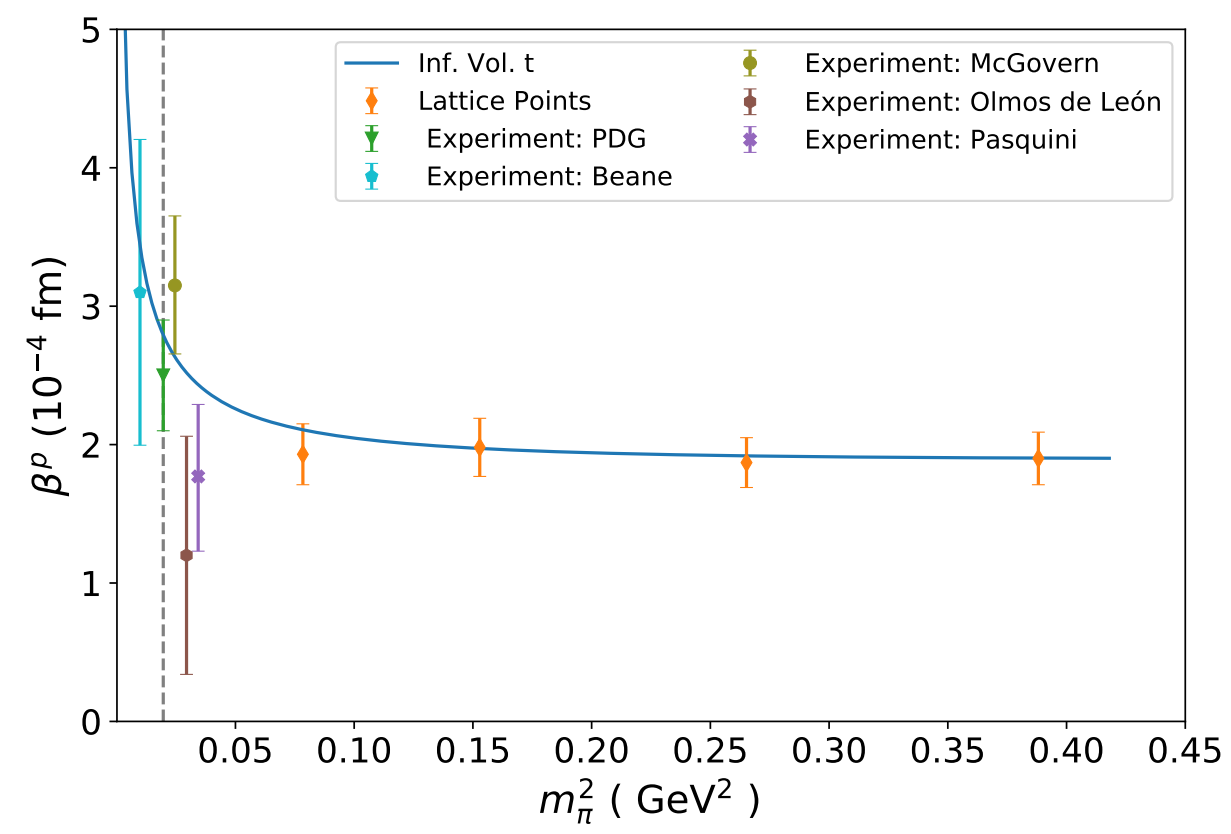

Figure 3. Experimental measurements of the magnetic polarisability of the proton, $\beta^{p}$, from the PDG [4], McGovern et al.[5], Beane et al.[24], Blanpied et al.[2], Olmos de León et al.[25], MacGibbon et al.[1] and Pasquini et al.[3] are offset for clarity and compared with the lattice QCD results produced herein. The finite-volume and electro-quenched corrected chiral extrapolation of Ref. [21] is also illustrated by the curve ( Inf. Vol. t ) for comparision with experiment.

of Adelaide. This research was undertaken with the assistance of resources from the National Computational Infrastructure (NCI). NCI resources were provided through the National Computational Merit Allocation Scheme, supported by the Australian Government through Grants No. LE190100021, LE160100051 and the University of Adelaide Partner Share. R.B. was supported by an Australian Government Research Training Program Scholarship. This research is supported by the Australian Research Council through Grants No. DP140103067, DP150103164, DP190102215 (D.B.L) and DP190100297 (W.K).

\section{References}

[1] B.E. MacGibbon, G. Garino, M.A. Lucas, A.M. Nathan, G. Feldman, B. Dolbilkin, Phys. Rev. C52, 2097 (1995), nucl-ex/9507001

[2] G. Blanpied, M. Blecher, A. Caracappa, R. Deininger, C. Djalali, G. Giordano, K. Hicks, S. Hoblit, M. Khandaker, O. Kistner et al., Phys. Rev. C64, 025203 (2001)

[3] B. Pasquini, P. Pedroni, S. Sconfietti (2019), 1903.07952

[4] M. Tanabashi et al. (Particle Data Group), Phys. Rev. D98, 030001 (2018)

[5] J.A. McGovern, D.R. Phillips, H.W. Griesshammer, Eur. Phys. J. A49, 12 (2013), 1210.4104

[6] J. Smit, J.C. Vink, Nucl. Phys. B286, 485 (1987)

[7] M. Burkardt, D.B. Leinweber, X.m. Jin, Phys. Lett. B385, 52 (1996), hep-ph/9604450 
[8] J.W. Lee, B.C. Tiburzi, Phys. Rev. D90, 074036 (2014), 1407. 8159

[9] G. Martinelli, G. Parisi, R. Petronzio, F. Rapuano, Phys. Lett. B116, 434 (1982)

[10] R. Bignell, J. Hall, W. Kamleh, D. Leinweber, M. Burkardt, Phys. Rev. D98, 034504 (2018), 1804.06574

[11] E. Chang, W. Detmold, K. Orginos, A. Parreno, M.J. Savage, B.C. Tiburzi, S.R. Beane, Phys. Rev. D92, 114502 (2015), 1506.05518

[12] C.W. Bernard, T. Draper, K. Olynyk, M. Rushton, Phys. Rev. Lett. 49, 1076 (1982)

[13] B.C. Tiburzi, S.O. Vayl, Phys. Rev. D87, 054507 (2013), 1210. 4464

[14] T. Primer, W. Kamleh, D. Leinweber, M. Burkardt, Phys. Rev. D89, 034508 (2014), 1307.1509

[15] C. Itzykson, J.B. Zuber, Quantum Field Theory, International Series in Pure and Applied Physics (McGraw-Hill , Inc., 1980)

[16] S. Gusken, Nucl. Phys. Proc. Suppl. 17, 361 (1990)

[17] F. Bruckmann, G. Endrodi, M. Giordano, S.D. Katz, T.G. Kovacs, F. Pittler, J. Wellnhofer, Phys. Rev. D96, 074506 (2017), 1705. 10210

[18] B.B. Brandt, G. Bali, G. Endrödi, B. Glässle, PoS LATTICE2015, 265 (2016), 1510.03899

[19] G.S. Bali, B.B. Brandt, G. Endrődi, B. Gläßle, Phys. Rev. D97, 034505 (2018), 1707.05600

[20] R. Bignell, W. Kamleh, D. Leinweber, Phys.Rev.D 100, 114518 (2019), 1910. 14244

[21] R. Bignell, W. Kamleh, D. Leinweber, Phys. Rev. D 101, 094502 (2020), 2002.07915

[22] W. Kamleh, R. Bignell, D.B. Leinweber, M. Burkardt, EPJ Web Conf. 175, 05018 (2018), 1711.07161

[23] B. Efron, SIAM Review 21, 460 (1979)

[24] S.R. Beane, M. Malheiro, J.A. McGovern, D.R. Phillips, U. van Kolck, Phys. Lett. B567, 200 (2003), [Erratum: Phys. Lett.B607,320(2005)], nucl-th/0209002

[25] V.O. de León et al., Eur. Phys. J. A10, 207 (2001) 